reviewed. Data were collected included baseline demographics, social factors including index of multiple deprivation (IMD), aetiology of liver disease, period of abstinence and assessment outcome (table 1).

Results 125 patients with a history of ArLD were referred for transplant assessment over the 3-year period. The majority (78\%) were male and most (39\%) were 50-59 years. Patients living in the most deprived quintile comprised just $17 \%$ of the group. In 50\% ArLD was the only aetiology listed, 27\% of patients also had hepatocellular carcinoma (HCC) and 28\% had a second, non-malignant, aetiology listed. Patients were divided into groups based on demographics, social factors, aetiology and period of abstinence and the outcome of the transplant assessment was recorded for each group. Table 1 illustrates the percentage of patients in each group listed for transplant. Psychological, social or substance misuse issues (including smoking) were cited as concern for listing in just 7 (5\%) of cases.

Conclusion Our data demonstrates that in patients with a history of ArLD referred to this unit for transplant assessment, there is no listing bias based on socioeconomic background. However, whilst ArLD occurs more often in lower socioeconomic groups, patients from IMD quintile 1 comprised just $17 \%$ of those referred, suggesting a potential referral bias. There was no clear preference for listing patients who had a second causal aetiology for their liver disease. Very few patients were not listed based purely on social/psychological factors. This data should encourage clinicians to refer patients with a history of ArLD for transplant assessment, regardless of their socioeconomic background.

\section{P093 TOLL-LIKE RECEPTOR 4 INHIBITION ACTS SYNERGISTICALLY WITH G-CSF TO PREVENT ORGAN INJURY AND INDUCE LIVER REGENERATION IN ACUTE- ON-CHRONIC LIVER FAILURE}

Cornelius Engelmann, Fausto Andreola*, Abeba Habtesion, Simone Novelli, Annarein JC Kerbert, Nathan Davies, Sofia Ferreira-Gonzalez, Stuart Forbes, Thomas Berg, Rajiv Jalan. Ildh - University College London; Charite - Universitaetsmedizin Berlin, Germany

\subsection{6/gutjnl-2021-BASL.101}

Background and Aims Acute-on-chronic liver failure (ACLF) is characterised by lack of regeneration. Granulocyte colony stimulating factor (G-CSF) carries pro-regenerative properties and has been shown to be of benefit in ACLF. However, the large trial of G-CSF (GRAFT study) in patients with ACLF showed no benefit and in certain groups mortality tended to be higher. This study was performed to define the mechanisms underlying the negative effect of G-CSF and determine whether its beneficial effect could be harnessed using a tolllike receptor 4 (TLR4) antagonist.

Method Two mouse models of ACLF were used: CCL4 $(0.5 \mathrm{mg} / \mathrm{ml}, 6 \mathrm{w})$ to induce chronic liver injury followed by LPS i.p. (Klebsiella, $4 \mathrm{mg} / \mathrm{kg})(\mathrm{n}=4-10)$ or Galactosamine (GalN) i. p. $(1000 \mathrm{mg} / \mathrm{kg})$ as a second hit $(\mathrm{n}=8)$. $1 \mathrm{~h}$ after, G-CSF $250 \mu \mathrm{g} / \mathrm{kg}$ s.c. and/or TLR4-inhibitor TAK-242 $10 \mathrm{mg} / \mathrm{kg}$ i.p. were injected and continued every $24 \mathrm{~h}$. The treatment duration was $24 \mathrm{~h}$ and $5 \mathrm{~d}$ in the LPS model and $48 \mathrm{~h}$ in the GalN model. Samples were stored and analysed for liver injury, inflammation, senescence and regeneration.

Results 6w CCL4 led to bridging fibrosis, TLR4 up-regulation and infiltration of G-CSFr expressing cells. LPS increased ALT levels, cell death $(\mathrm{TUNEL}+)$, enhanced hepatic infiltration of neutrophils (Ly6G+), macrophages $(\mathrm{F} 4 / 80+)$ and TNFa. GCSF increased the $48 \mathrm{~h}$ mortality from $0 \%$ to $66 \%$, aggravated liver inflammation with macrophage and NK cell (CD45+, CD49b+,CD3-,CD19-) infiltration and IL6 expression. G-CSF +TAK-242 reduced the mortality to $0 \%$, abrogated the liver injury (TUNEL) and liver inflammation (macrophages, neutrophils, TNFa, IL6) significantly. In the second model, GalN also induced a significant liver injury. Treatment with G-CSF +TAK-242 was significantly more effective than the individual therapies. G-CSF+TAK-242 was associated with increased liver regeneration evidenced by increased tissue expression of pSTAT3 and BCL2. CCL4+LPS induced a p53 and p16dependent cell cycle arrest and lack of proliferation (CyclinA) in hepatocytes. G-CSF+TAK-242 mitigated senescence and significantly increased the rate of CyclinA expressing hepatocytes (figure) suggesting enhanced liver regeneration.

Conclusion The present study shows that G-CSF is deleterious in LPS-associated ACLF through further activation of inflammatory pathways and immune cell infiltration. TLR4 inhibition with TAK-242 prevented G-CSF driven tissue injury and induced liver regeneration showing evidence of synergy between the two molecules thereby providing a novel therapeutic strategy for ACLF patients.

\section{P094 POTENTIAL MECHANISMS UNDERLYING THE PROTECTIVE EFFECT OF LONG-TERM ALBUMIN INFUSION IN CIRRHOSIS} ${ }^{1,2}$ Qianwen Zhao, ${ }^{1}$ Alexandra Phillips*, ${ }^{1}$ Abeba Habtesion, ${ }^{1}$ Fausto Andreola,
${ }^{1}$ Nathan Davies, ${ }^{1} J a n e$ MacNaughtan, 'Rajiv Jalan. 'University College London, Institute for
Liver and Digestive Health, London, UK; ' ${ }^{2}$ Department of Gastroenterology and Hepatology,
West China Hospital, Sichuan University, China

\subsection{6/gutjnl-2021-BASL.102}

Background Long-term albumin administration has shown a reduction in mortality in hepatic decompensation, however the mechanism of this is unclear. The aims of this study were to determine (i) whether analbuminaemic (NAR) rats have greater liver injury and sensitivity to LPS (ii) whether albumin infusion is protective in NAR rats (iii) the impact of analbuminaemia and albumin infusion on the gut-liver interface and hepatic TLR4 signalling.

Methods 10 treatment groups of NAR and SD rats were studied $(n=4-7)$; Naïve, cirrhosis (4-w after bile duct ligation (BDL)) and ACLF models (induced by lipopolysaccharide (LPS) to BDL) were studied. BDL groups: \pm LPS, \pm albumin infusion (1.5 g/kg i.p. for 2 weeks). Markers of liver injury: plasma ALT level and TUNEL staining; markers of gut integrity and permeability: DAB immunohistochemistry ZO-1 expression in ileum tissue; Hepatic TLR4 immunohistochemistry and related pathway genes RT2 PCR profiler were studied.

Results Liver injury: ALT levels and TUNEL positive areas were significantly higher in NAR compared with SD rats $(\mathrm{p}=$ 0.01 and $\mathrm{p}=0.01)$, which were corrected with albumin infusion $(\mathrm{p}=0.02, \mathrm{p}=0.047)$. Effect of LPS administration: coma-free survival was higher in SD rats than NAR rats $(80 \%$ vs $40 \%$ ). Effect of albumin administration: Administration of albumin to BDL rats reduced severity of liver injury and mortality after LPS administration [p $=0.001 ; 40 \%$ vs $100 \%$, p $=0.04]$. Markers of gut permeability: In NAR rats, the histopathological examination of the ileum and colon revealed a reduction in ZO-1 expression, which was restored with 